\title{
Myeloid cell-specific expression of Ship1 regulates IL-12 production and immunity to helminth infection
}

\author{
S Hadidi ${ }^{1,7}$, F Antignano ${ }^{1,7}$, MR Hughes ${ }^{1,7}$, SKH Wang ${ }^{1}$, K Snyder ${ }^{1}$, GM Sammis ${ }^{2}$, WG Kerr ${ }^{3,4}$, \\ KM McNagny ${ }^{1,5}$ and C Zaph ${ }^{1,6}$
}

Helminth infection leads to the local proliferation and accumulation of macrophages in tissues. However, the function of macrophages during helminth infection remains unclear. SH2-containing inositol 5'-phosphatase 1 (Ship1, Inpp5d) is a lipid phosphatase that has been shown to play a critical role in macrophage function. Here, we identify a critical role for Ship1 in the negative regulation of interleukin (IL)-12/23p40 production by macrophages during infection with the intestinal helminth parasite Trichuris muris. Mice with myeloid cell-specific deletion of Ship1 (Ship $1^{\Delta \mathrm{LysM}}$ mice) develop a non-protective T-helper type $1 \mathrm{cell}$ response and fail to expel parasites. Ship1-deficient macrophages produce heightened levels of IL-12/23p40 in vitro and in vivo and antibody blockade of IL-12/23p40 renders Ship $1^{\Delta L y s M}$ mice resistant to Trichuris infection. Our results identify a critical role for the negative regulation of IL-12/23p40 production by macrophages in the development of a protective $\mathrm{T}_{\mathrm{H}} 2$ cell response.

\section{INTRODUCTION}

Protective immunity against helminth infection is associated with the development of a type 2 immune response, characterized by activation and expansion of $\mathrm{CD} 4^{+}$T-helper type 2 $\left(\mathrm{T}_{\mathrm{H}} 2\right)$ cells that produce cytokines such as interleukin (IL)-4 and IL-13, induction of IgG1- and IgE-secreting plasma cells, as well as increased numbers of macrophages, eosinophils, mast cells, and basophils. ${ }^{1}$ Elevated levels of IL- 4 and IL-13 result in increased intestinal permeability and enhanced mucus and protein secretion by goblet cells, and increased smooth-muscle cell contractility. ${ }^{2-6}$ In contrast, susceptibility to helminth infection is associated with a non-protective T-helper type $1\left(\mathrm{~T}_{\mathrm{H}} 1\right)$ response that results in high levels of IL-12, IL-18, and interferon (IFN) $\gamma$ that permits chronic infection. ${ }^{7,8}$ As dysregulated $\mathrm{T}_{\mathrm{H}} 2$ cell responses are associated with a variety of inflammatory diseases including asthma and allergies, a better understanding of the cellular and molecular mechanisms that control $\mathrm{T}_{\mathrm{H}} 2$ cell development would be likely to reveal novel targets for therapeutic intervention in treating these diseases.
Macrophages are a highly plastic and functionally diverse subset of cells that play a key role in virtually all aspects of immune responses. ${ }^{9}$ Macrophages act as initiators of immune responses by presenting antigen to $T$ cells, regulators of immunity by producing immunoregulatory cytokines such as IL-10 or TGF $\beta$, and immune effectors through their phagocytic uptake and killing of bacterial, fungal, and protozoal pathogens by nitric oxide and reactive oxygen species-mediated processes. ${ }^{10,11}$ However, the detailed molecular mechanisms that control the differentiation and function of various macrophage subsets, particularly during $\mathrm{T}_{\mathrm{H}} 1$ and $\mathrm{T}_{\mathrm{H}} 2$ responses, remain unclear.

The role of macrophages during helminth infection is controversial. Macrophage numbers increase significantly in the tissues following infection with a variety of helminth parasites, ${ }^{12-15}$ and recent data in the peritoneal cavity suggest that this may be due to local IL-4-dependent proliferation rather than recruitment of precursors from the blood. ${ }^{16}$ Despite this expansion, it has proven difficult to show a clear functional link between their presence and resistance to helminth

\footnotetext{
${ }^{1}$ The Biomedical Research Centre, University of British Columbia, Vancouver, British Columbia, Canada. ${ }^{2}$ Department of Chemistry, University of British Columbia, Vancouver, British Columbia, Canada. ${ }^{3}$ Department of Microbiology and Immunology, SUNY Upstate Medical University, Syracuse, New York, USA. ${ }^{4}$ Department of Pediatrics, SUNY Upstate Medical University, Syracuse, New York, USA. ${ }^{5}$ Department of Medical Genetics, University of British Columbia, Vancouver, British Columbia, Canada. ${ }^{6}$ Department of Pathology and Laboratory Medicine, University of British Columbia, Vancouver, British Columbia, Canada. ${ }^{7}$ These authors contributed equally to this work.. Correspondence: C Zaph (colby@brc.ubc.ca) 
infection. Macrophages associated with anti-helminth $\mathrm{T}_{\mathrm{H}^{2}} 2$ cell responses adopt an alternative differentiation state, which has been termed M2. ${ }^{17,18}$ The M2 macrophage phenotype is characterized by the expression of a set of genes including arginase 1 (Arg1). ${ }^{19}$ Increased Arg1 expression is associated with suppression of adaptive immune responses by depleting local concentrations of arginine, an essential amino acid. ${ }^{20} \mathrm{M} 2$ polarization is mediated by IL-4 or IL-13, which both signal through the IL-4R $\alpha$ chain, and accordingly IL-4R $\alpha$-deficient macrophages fail to acquire an M2 phenotype. Interestingly, mice with a macrophage-specific deletion of IL-4R $\alpha$ develop normal protective $\mathrm{T}_{\mathrm{H}} 2$ cell-dependent immune responses following infection with the parasitic helminths Nippostrongylus brasiliensis $^{21}$ or Trichinella spiralis. ${ }^{22}$ In addition, mice treated with Arg1 inhibitors or mice deficient in Arg1 are not impaired in their ability to clear many helminth parasite infections. ${ }^{23,24}$ Furthermore, depletion of macrophages in resistant C57BL/6 mice during infection with the intestinal helminth parasite Trichuris muris ${ }^{25}$ has no effect on the development of protective responses and parasite expulsion. ${ }^{26}$ In summary, while macrophage differentiation and accumulation in infected tissues is a hallmark of helminth infections, the role of macrophages in the development of protective $\mathrm{T}_{\mathrm{H}} 2$ cell-dependent immunity is not clear.

SH2-containing inositol $5^{\prime}$-phosphatase 1 (Ship1, Inpp5d) is a lipid phosphatase that hydrolyses the $5^{\prime}$-phosphate of phosphatidylinositol-3,4,5-trisphosphate $(\mathrm{PI}(3,4,5) \mathrm{P} 3)$ to produce $\mathrm{PI}(3,4) \mathrm{P} 2$, thereby negatively regulating the phosphoinositide 3-kinase (PI3K) pathway. Ship1 ${ }^{-/}$mice have multiple immunological defects, including a myeloproliferative disorder, characterized by overproduction of granulocytes, mast cells, ${ }^{27}$ macrophages, ${ }^{28}$ and dendritic cells (DCs), ${ }^{29,30}$ and elevated immunosuppressive myeloid-derived suppressor cells ${ }^{29}$ and regulatory T cells. ${ }^{31,32}$ In addition, $\mathrm{CD} 4{ }^{+} \mathrm{T}$ cells from Ship $1^{-/-}$ mice produce heightened levels of the $\mathrm{T}_{\mathrm{H}} 2$ cell-associated cytokines IL- 4 and IL-13. ${ }^{33}$ Peritoneal and alveolar macrophages from naïve Ship1 $1^{-/-}$mice display an M2 phenotype, expressing high levels of Arg1. ${ }^{34}$ In contrast, Ship $1^{-/-}$bone marrow-derived macrophages (BMMacs) produce heightened levels of IL- 6 and tumor necrosis factor- $\alpha$ in response to lipopolysaccharide (LPS) stimulation, fail to undergo LPSinduced tolerance, and Ship $1^{-1-}$ mice are highly sensitive to endotoxic shock. ${ }^{35}$

On the basis of these studies, we hypothesized that Ship1-1mice would display heightened $\mathrm{T}_{\mathrm{H}} 2$ cell responses and increased resistance to helminth infection. Here we report that in contrast to our expectations, following infection with Trichuris Ship $1^{-/-}$ mice fail to develop a polarized protective $\mathrm{T}_{\mathrm{H}} 2$ cell response and are susceptible to infection. Furthermore, we have employed myeloid cell-specific Ship1-deficient mice (Ship $1^{\Delta \text { LysM }}$ mice) to show that dysregulated macrophage function impacts the development of a protective anti-helminth response. We show that in normal macrophages, Ship 1 is required to limit IL-12 production by macrophages and that this in turn is required for the development of a protective $\mathrm{T}_{\mathrm{H}} 2$ cell response. Taken together, our results reveal a surprising and previously unappre- ciated role for Ship1 in the development of protective immunity to helminth infection.

\section{RESULTS}

\section{Ship $1^{-1-}$ mice are susceptible to infection with Trichuris}

It has previously been demonstrated that naïve Ship $1^{-/-}$mice are biased towards type 2 immune responses, with increased levels of IgE, heightened production of type 2 cytokines by T cells and basophils, and the presence of M2 macrophages. ${ }^{33-35}$
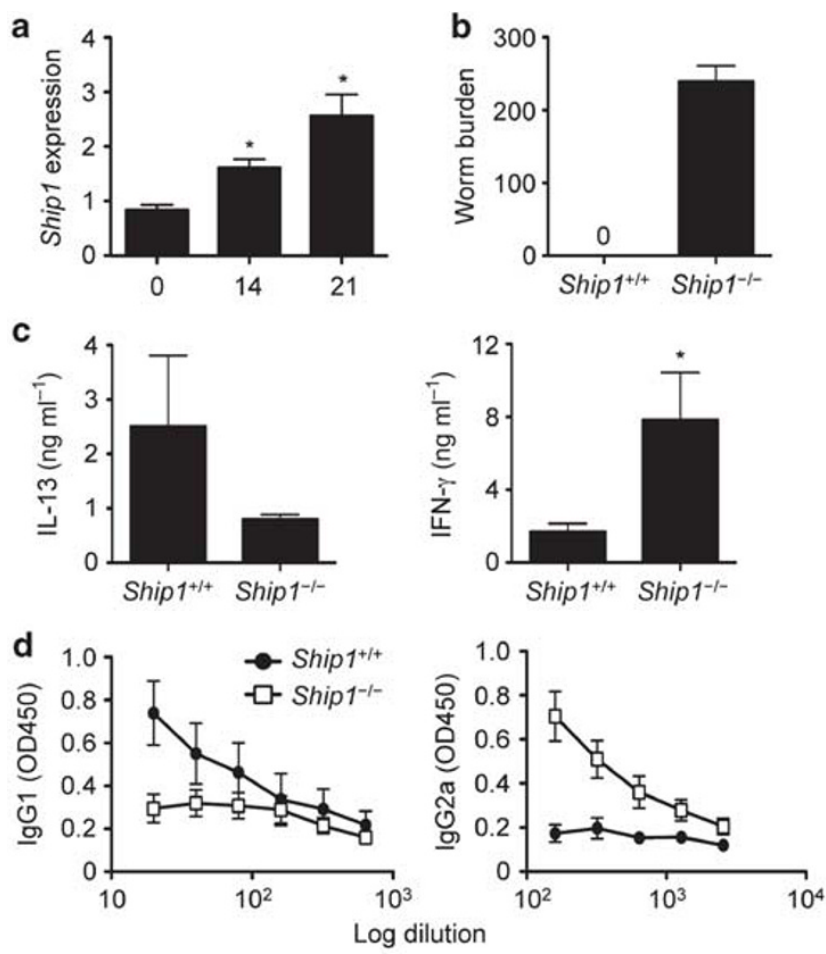

e
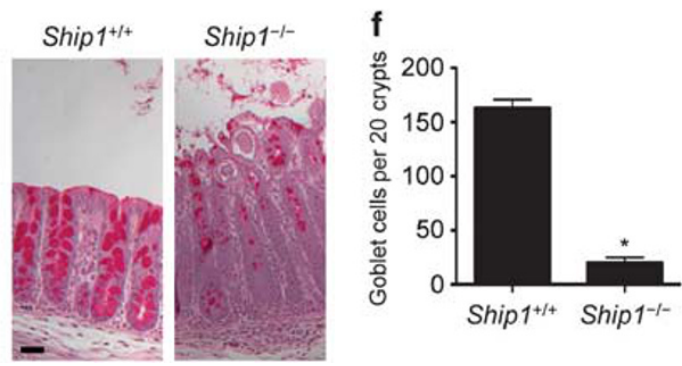

Figure 1 SH2-containing inositol 5'-phosphatase 1 (Ship1) is required for immunity to Trichuris infection. (a) Ship1 mRNA expression determined by quantitative polymerase chain reaction (qPCR) from intestinal tissue harvested from naïve (0) control (Ship $\left.1^{+/+}\right)$mice or control mice at days 14 and 21 post-infection. (b) Control (Ship $1^{+/+}$) and knockout (Ship $1^{-/-}$) mice were infected with 250 Trichuris eggs and killed on day 21 post-infection. Worm burdens were determined microscopically. (c) Mesenteric lymph node (LN) cells were cultured with Trichuris antigen for $72 \mathrm{~h}$ and cell-free supernatants were analyzed by enzyme-linked immunosorbent assay (ELISA) for the expression of interleukin (IL)-13 and interferon (IFN)- $\gamma$. (d) Trichuris-specific serum immunoglobulin responses were determined by ELISA. (e) Cecal sections were stained with periodic acid-Schiff's stain. (f) Goblet cells were enumerated microscopically. Data are mean \pm s.e.m. and are from one of three independent experiments ( $n=4-5$ per experiment). ${ }^{*} P<0.05$. 
On the basis of these previous studies, we hypothesized that Ship $1^{-1-}$ mice would display increased resistance to infection with the helminth parasite Trichuris. However, following infection with Trichuris, genetically resistant C57BL/6 mice display increased expression of Ship $1 \mathrm{mRNA}$ in the intestine (Figure 1a), suggesting that Ship1 expression is associated with immunity to infection. Consistent with this, Ship $1^{-1-}$ mice were more susceptible to Trichuris infection, failing to expel worms by day 21 (Figure 1b). Restimulation of mesenteric lymph node ( $\mathrm{mLN}$ ) cells isolated from infected Ship $1^{-/-}$mice with Trichuris antigen resulted in decreased levels of IL-13 and heightened levels of IFN- $\gamma$ when compared with Ship $1^{+/+}$mice (Figure 1c). Consistent with this skewed immune response, infected Ship $1^{-1-}$ mice also displayed lower levels of Trichurisspecific serum IgG1 and higher IgG2a than infected Ship1 $1^{+/+}$ mice (Figure 1d). Although naïve Ship $1^{+/+}$and Ship $1^{-/-}$ mice were indistinguishable histologically (data not shown), infected Ship $1^{-1-}$ mice displayed increased inflammation, loss of crypt architecture, and lack of goblet cells compared with control mice (Figure 1e). While goblet cell numbers were equivalent between naïve Ship $1^{+/+}$and Ship $1^{-/-}$mice (data not shown), following Trichuris infection there were significantly fewer goblet cells in the intestines of Ship1 ${ }^{-/-}$mice (Figure 1f). Thus, Ship $1^{-/-}$mice fail to generate a protective antigen-specific $\mathrm{T}_{\mathrm{H}} 2$ cell response during infection with Trichuris, developing instead a polarized non-protective $\mathrm{T}_{\mathrm{H}} 1$ cell response.

\section{Ship ${ }^{\Delta \text { LysM }}$ mice are susceptible to infection with Trichuris}

We sought to test whether myeloid cell-specific Ship1 expression was required for resistance to intestinal helminth infection. We generated mice with a specific deletion of Ship1 in myeloid cells, including macrophages and neutrophils (Ship $1^{\Delta \mathrm{LysM}}$ mice) by crossing Ship $1^{\mathrm{F} / \mathrm{F}}$ mice with $L y s M$-cre transgenic mice. ${ }^{36-38}$ Ship $1^{\mathrm{F} / \mathrm{F}}$ or Ship $1^{\Delta \mathrm{LysM}}$ mice were infected with Trichuris and analyzed on days 21 and 32 post-infection. Strikingly, Ship $1^{\Delta \mathrm{LysM}}$ mice failed to expel worms by day 32 , while control Ship $1^{\mathrm{F} / \mathrm{F}}$ mice had cleared all parasites by day 21 (Figure 2a). Susceptibility to infection in Ship $1^{\Delta \text { LysM }}$ mice was associated with increased levels of IFN- $\gamma$ produced by mLN cells (Figure $2 \mathbf{b}$ ) and Ifng gene expression in the intestine (Figure $2 \mathrm{c}$ ). While we observed no difference in IL-4, IL-5, and IL-13 production by restimulated
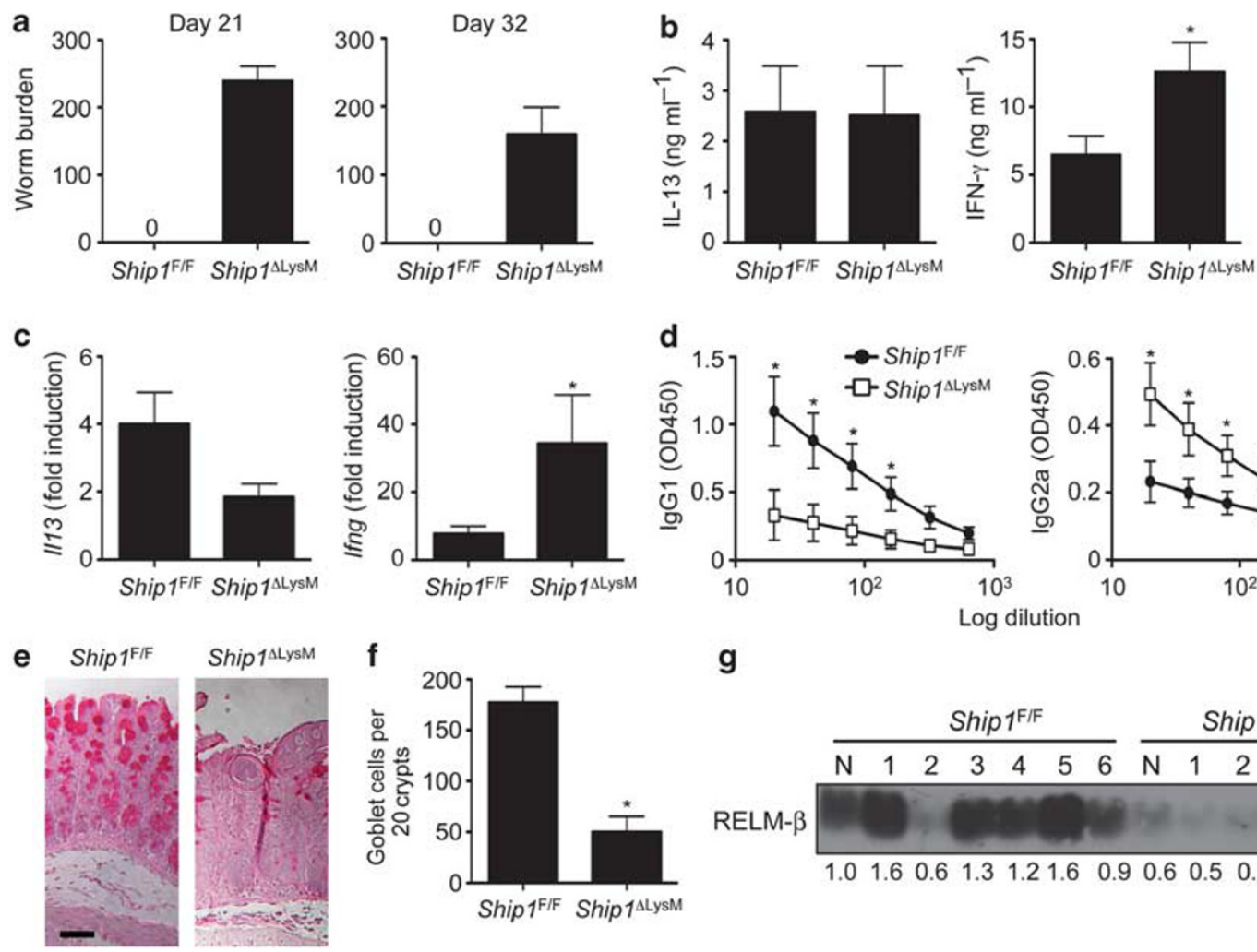

g

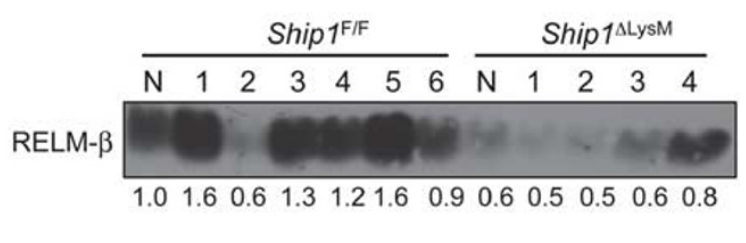

Figure 2 Myeloid cell-intrinsic expression of SH2-containing inositol 5'-phosphatase 1 (Ship1) is required for immunity to Trichuris infection. Control $\left(\right.$ Ship $\left.^{\mathrm{F} / \mathrm{F}}\right)$ and knockout (Ship $1^{\mathrm{ALySM}}$ ) mice were infected with 250 Trichuris eggs and killed on day 21 or day 32 post-infection. (a) Worm burdens in the cecum were determined microscopically. (b) Mesenteric lymph node ( $L N)$ cells were cultured with Trichuris antigen for $72 \mathrm{~h}$ and cell-free supernatants were analyzed by enzyme-linked immunosorbent assay (ELISA) for the expression of interleukin (IL)-13 and interferon (IFN)- $\gamma$. (c) I/13 and Ifng mRNA expression in the intestinal tissue from Trichuris-infected Ship $1^{\mathrm{F} / \mathrm{F}}$ and Ship $1^{\Delta \mathrm{LysM}}$ mice was examined by quantitative polymerase chain reaction (qPCR). (d) Trichuris-specific serum immunoglobulin responses were determined by ELISA. (e) Cecal sections were stained with periodic acid-Schiff's stain. Bar $=25 \mu \mathrm{m}$. (f) Goblet cells were enumerated microscopically. (g) Immunoblot for resistin-like molecule (RELM) $\beta$ secreted into the gut lumen. Densitometry values relative to naïve control mice $\left(\mathrm{N}\right.$, Ship $\left.{ }^{\mathrm{F} / \mathrm{F}}\right)$ are shown. Data in column graphs are the mean \pm S.e.m. and are from one experiment and are representative of three independent experiments $\left(n=4-5\right.$ per experiment). ${ }^{*} P<0.05$. 
mLN cells between Ship $1^{\mathrm{F} / \mathrm{F}}$ or Ship $1^{\Delta \mathrm{LysM}}$ mice (Figure $2 \mathbf{b}$ and Supplementary Figure 1A), we did detect decreased levels of intestinal Il13 mRNA in Ship $1^{\Delta \mathrm{LysM}}$ mice (Figure 2c), but not Il4 or Il5 mRNA (Supplementary Figure 1B). Consistent with increased IFN- $\gamma$ production, we also detected decreased levels of Trichuris-specific IgG1 and increased levels of IgG2a in the serum (Figure 2d). Furthermore, histological analyses revealed heightened inflammation in infected Ship $1^{\Delta \mathrm{LysM}}$ mice, with loss of crypt structure and elevated inflammatory cell infiltrates (Figure 2e), but a failure to induce goblet cell hyperplasia (Figure 2f) and expression and secretion of resistin-like molecule (RELM) $\beta$ (Figure 2g). Thus, expression of Ship1 in myeloid cells is required for all key hallmarks of protective $\mathrm{T}_{\mathrm{H}} 2$ cell-dependent immunity to Trichuris. As macrophages have been shown to play a role in several helminth infections, ${ }^{12-16}$ we focused our studies on macrophages.

\section{Ship1 controls expression of $\operatorname{Arg} 1$ in the peritoneal cavity but not the intestine}

On the basis of previous studies using conventional Ship $1^{-/-}$ mice, ${ }^{34,39}$ we hypothesized that macrophages from Ship $1^{\Delta \mathrm{LysM}}$ mice would express high levels of the M2 macrophage marker Arg1. ${ }^{19}$ Peritoneal macrophages isolated from Ship $1^{\Delta \mathrm{LysM}}$ mice displayed high levels of Arg1 as measured by mRNA expression (Figure 3a), protein production (Figure 3b), and enzymatic activity (Figure 3c). However, we observed no difference in the levels of Arg1 mRNA in the intestine of Ship $1^{\mathrm{F} / \mathrm{F}}$ or Ship $1^{\Delta \mathrm{LysM}}$ mice (Figure 3d). Expression of Arg1 is upregulated following infection with several helminth parasites, including Heligmosomoides polygyrus, N. brasiliensis, and Schistosoma mansoni. ${ }^{23,40,41}$ A recent study has demonstrated that during Trichuris infection, there is a mild increase in the frequency of Arg1-expressing cells in the intestine, but that Arg1 is not required for immunity to infection. ${ }^{24}$ While we observed an infection-induced increase in Arg1 gene expression in the intestine, we did not observe any differences between Trichurisinfected Ship $1^{\mathrm{F} / \mathrm{F}}$ and Ship $1^{\mathrm{LLysM}}$ mice (Figure 3d). Consistent with these results, treatment of mice with the Arg1 inhibitor (S)-(2-boronoethyl)-L-cysteine (BEC) had no effect on the outcome of Trichuris infection in Ship $1^{\mathrm{F} / \mathrm{F}}$ or Ship $1^{\Delta \mathrm{LysM}}$ mice (data not shown). We also failed to observe any differences in the expression of another M2 cell marker, RELM $\alpha$ (Retnla) (data not shown). However, we did observe significantly increased expression of inducible nitric oxide synthase (Nos2), a marker for M1 macrophages, consistent with the heightened levels of IFN- $\gamma$ and IL-12 observed in the intestines of Ship $1^{\Delta \mathrm{LysM}}$ mice (Figure 3e). Thus, although Ship1 controls Arg1 expression in peritoneal macrophages, it has no effect on Arg1 expression in the intestine. Furthermore, we conclude that the susceptibility of Ship $1^{\Delta \mathrm{LysM}}$ mice to Trichuris infection is not due to the presence of dysregulated M2 macrophages or expression of Arg1.

\section{Antibody blockade of IFN- $\gamma$ in Trichuris-infected Ship ${ }^{\Delta \text { LysM }}$ mice promotes resistance}

As we observed heightened levels of IFN- $\gamma$ in infected Ship $1^{\Delta \mathrm{LysM}}$ mice (Figure $\mathbf{2 b}$ ), we next asked whether antibody blockade
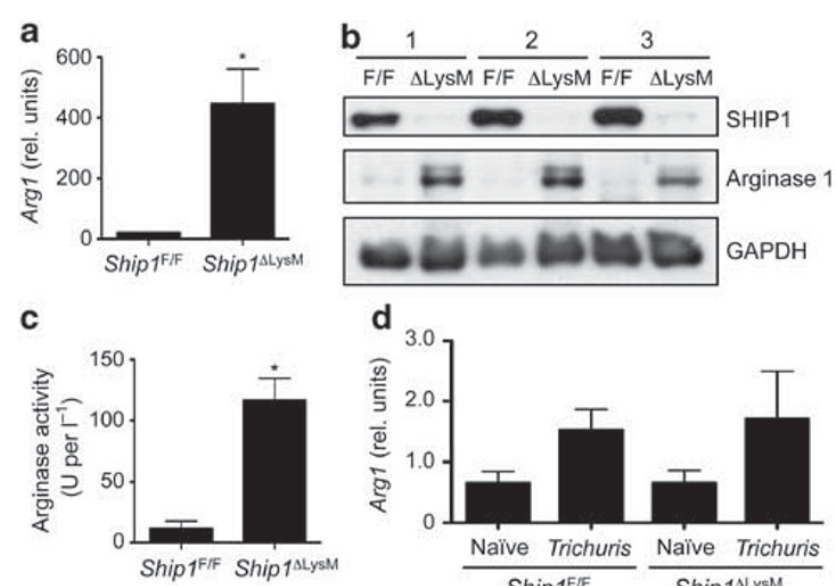

d
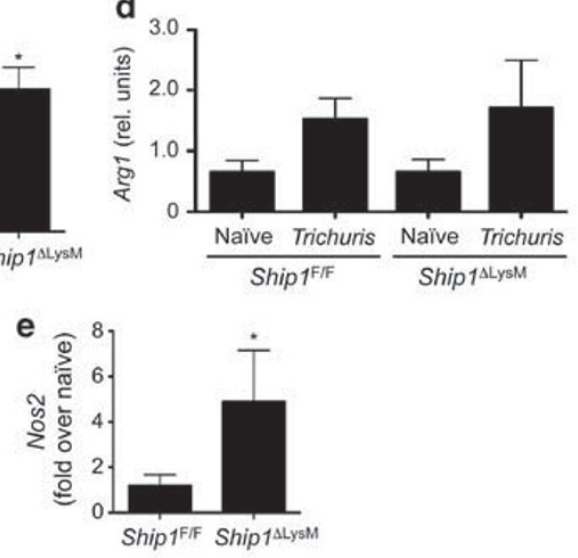

Figure 3 Myeloid cell-intrinsic expression of $\mathrm{SH}$ 2-containing inositol 5'-phosphatase 1 (Ship1) controls arginase 1 (Arg1) expression in the peritoneum, but not the intestine. (a) Arg1 mRNA expression in peritoneal macrophages isolated from control (Ship $1^{\mathrm{F} / F}$ ) and knockout (Ship $1^{\Delta \mathrm{LysM}}$ ) mice was examined by quantitative polymerase chain reaction (qPCR). (b) Arginase 1 protein levels were analyzed in peritoneal macrophages from Ship $1^{\mathrm{F} / \mathrm{F}}$ or Ship $1^{\text {LLysM }}$ mice by immunoblotting. (c) Arginase 1 activity in peritoneal macrophages from Ship $1^{\mathrm{F} / \mathrm{F}}$ and Ship $1^{\mathrm{LLysM}}$ mice was determined using a colorimetric assay. (d) Expression of Arg1 mRNA in the intestinal tissue from Ship $1^{\mathrm{F} / \mathrm{F}}$ and Ship ${ }^{\mathrm{LLysM}}$ mice was examined by qPCR. (e) Ship $1^{\mathrm{F} / \mathrm{F}}$ and Ship $1^{\Delta \mathrm{LysM}}$ mice were infected with 250 Trichuris eggs and killed on day 21 post-infection. Expression of Nos2 mRNA in the intestinal tissue was examined by qPCR. Data are mean \pm s.e.m. from one experiment and are representative of three independent experiments ( $n=4$ mice per experiment). ${ }^{\star} P<0.05$.

of IFN- $\gamma$ would render Ship $1^{\Delta \mathrm{LysM}}$ mice resistant to infection. Treatment with $\alpha$-IFN- $\gamma$ antibody reversed the susceptibility to Trichuris infection in Ship $1^{\Delta \mathrm{LysM}}$ mice, with a significant reduction in worm burden in antibody-treated mice (Figure 4a). Antibody treatment also resulted in increased IL-13 and decreased IFN- $\gamma$ production by mLN cells (Figure $4 \mathbf{b}$ ), as well as reduced levels of Trichuris-specific IgG2a in the serum of infected mice (Figure 4c). Increased resistance was associated with increased numbers of goblet cells and increased expression and secretion of RELM $\beta$ (Figure $4 \mathbf{d}$ and $\mathbf{e}$ ). Thus, these results suggest that macrophage-specific expression of Ship 1 is not required for resistance to Trichuris infection when nonprotective $\mathrm{T}_{\mathrm{H}} 1$ cell responses are blocked.

\section{Ship $1^{-/-}$macrophages produce increased levels of IL-12p40 in vitro and in vivo}

Consistent with the high levels of IFN- $\gamma$ observed in Trichurisinfected Ship $1^{\Delta \mathrm{LysM}}$ mice, mLN cells isolated from infected Ship $1^{\Delta \mathrm{LysM}}$ mice cultured in either medium alone or in the presence of Trichuris antigen produced significantly increased levels 

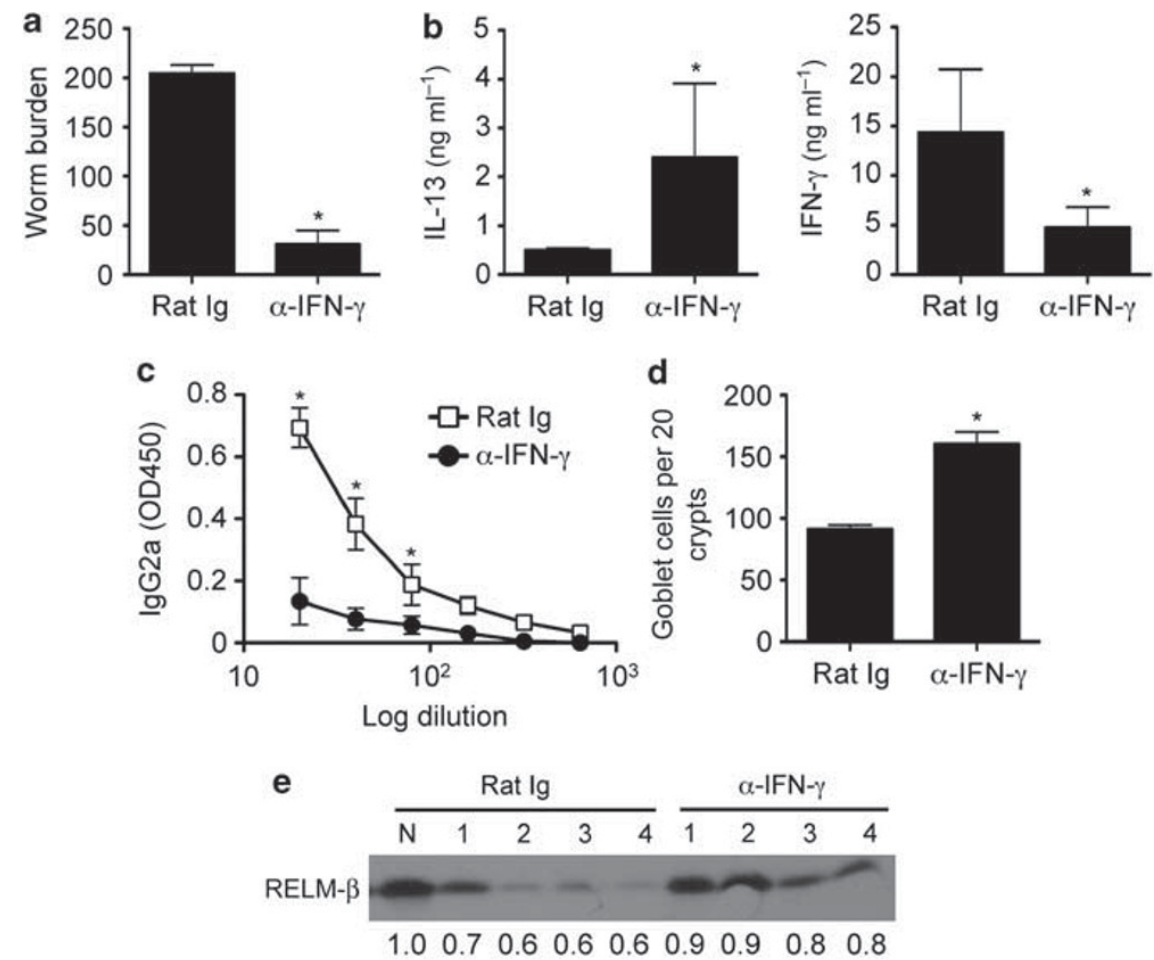

Figure 4 Blockade of interferon (IFN)- $\gamma$ renders Ship $1^{\Delta \mathrm{L} y \mathrm{sM}}$ mice resistant to infection with Trichuris. Ship $1^{\Delta \mathrm{LysM}}$ mice were infected with 250 Trichuris eggs, treated intraperitoneally (i.p.) with $500 \mu \mathrm{g}$ of either control rat Ig or anti-IFN $\gamma$ (XMG-1.2) every 4 days from day 4 to 20 and killed on day 21 post-infection. (a) Worm burdens were determined microscopically. (b) Mesenteric lymph node (LN) cells were cultured with Trichuris antigen for $72 \mathrm{~h}$ and cell-free supernatants were analyzed by enzyme-linked immunosorbent assay (ELISA) for the expression of interleukin (IL)-13 and IFN- $\gamma$.

(c) Trichuris-specific serum immunoglobulin responses were determined by ELISA. (d) Goblet cells were enumerated microscopically. (e) Immunoblot (with relative densitometry values) for resistin-like molecule (RELM) $\beta$ secreted into the gut lumen. Data are mean \pm s.e. $m$. from one experiment and are representative of two independent experiments ( $n=4-5$ per experiment). ${ }^{*} P<0.05$. Ship1, SH2-containing inositol $5^{\prime}$-phosphatase 1.

of IL-12/23p40 (Figure 5a). We also observed heightened levels of $I l 12 a$ and $I l 12 b$ gene expression in the intestine of Ship $1^{\Delta \mathrm{LysM}}$ mice following Trichuris infection (Figure 5b). Finally, LPS stimulation of BMMacs derived from Ship $1^{\mathrm{F} / \mathrm{F}}$ or Ship $1^{\Delta \mathrm{LysM}}$ mice revealed increased levels of IL-12/23p40 production from Ship1-deficient macrophages (Figure 5c). Thus, Ship1 is intrinsically required to limit the production of IL-12/23p 40 by macrophages.

\section{Antibody blockade of IL-12p40 in Trichuris-infected Ship $1^{\Delta \mathrm{LysM}}$ mice promotes resistance}

To test directly whether dysregulated production of IL-12 was responsible for the enhanced susceptibility of Ship $1^{\Delta \mathrm{LysM}}$ mice to Trichuris infection, we treated Ship ${ }^{\Delta \mathrm{LysM}}$ mice with a blocking antibody against IL-12/23p40 during Trichuris infection. Blockade of IL-12/23p40 in Ship ${ }^{\Delta \mathrm{LysM}}$ mice resulted in increased resistance to infection, characterized by reduced worm burden (Figure 6a), heightened IL-13, and decreased IFN- $\gamma$ production by restimulated mLN cells (Figure $6 \mathbf{b}$ ), and gene expression in the intestine (Figure 6c). In addition, elevated serum levels of Trichuris-specific IgG1 (Figure 6d), increased goblet cell hyperplasia (Figure $6 \mathbf{e}$ and $\mathbf{f}$ ), and elevated expression and secretion of RELM $\beta$ into the intestinal lumen (Figure 6g) were all observed after anti-IL-12/23p40 treatment.
Taken together, these results demonstrate that Ship1-dependent negative regulation of IL-12/23p 40 production by macrophages is a key step required for the development of protective $\mathrm{T}_{\mathrm{H}} 2$ cell immunity to helminth infection.

\section{DISCUSSION}

Here we demonstrate a role for macrophages in determining the adaptive immune response during helminth infection. In the absence of Ship1, macrophages produce increased levels of IL-12 and promote non-protective $\mathrm{T}_{\mathrm{H}} 1$ cell responses during infection with Trichuris. Blockade of IL-12/23p40 in Ship $1^{\text {LLysM }}$ mice leads to the restoration of a $\mathrm{T}_{\mathrm{H}} 2$ cell response and immunity to infection. Thus, although macrophages are not required for the development of anti-helminth immune responses, dysregulated macrophage functions can dramatically impact the outcome of infection. This study highlights a specific and critical role for macrophages during intestinal immune responses.

Conventional Ship $1^{-1-}$ mice display prominent macrophage skewing towards an M2 phenotype with alveolar and peritoneal macrophages expressing high levels of $\operatorname{Arg} 1 .{ }^{34}$ While we also find that peritoneal macrophages from Ship $1^{\Delta \mathrm{LysM}}$ mice expressed high levels of Arg1, we were surprised to find that intestinal tissues from Ship $1^{\Delta \mathrm{LysM}}$ mice do not display heightened expression of Arg1. Consistent with these results, a recent 

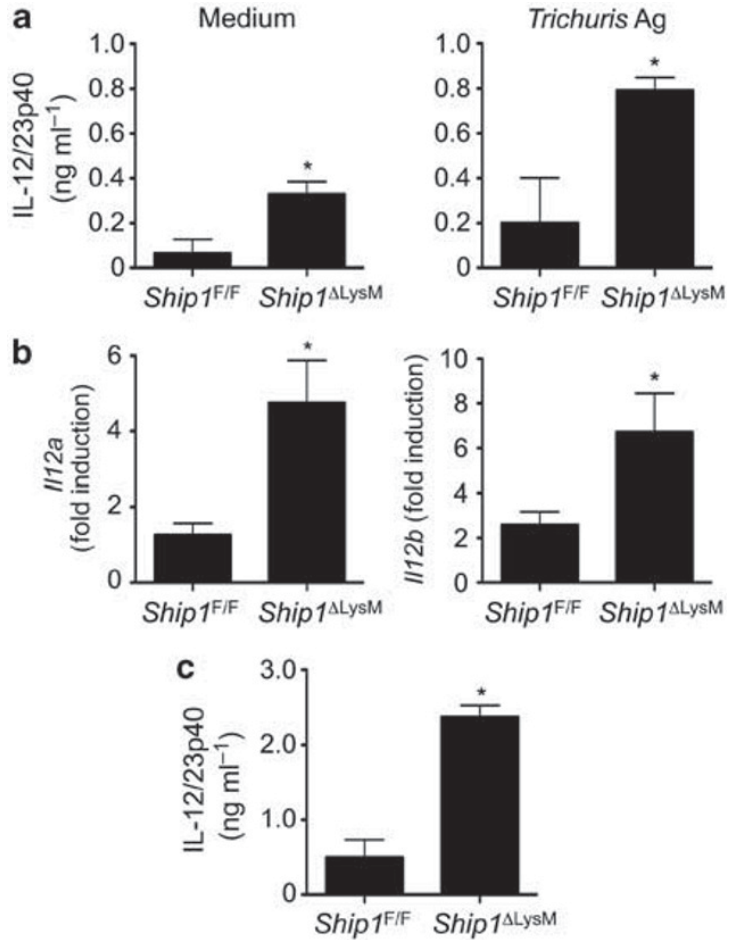

Figure 5 Macrophage-intrinsic expression of $\mathrm{SH} 2$-containing inositol 5 '-phosphatase 1 (Ship1) is required for negative regulation of interleukin (IL)-12/23p40 production in vitro and in vivo. (a) Ship $1^{\mathrm{F} / \mathrm{F}}$ and Ship $1^{\mathrm{LLysM}}$ mice were infected with 250 Trichuris eggs and killed on day 21 postinfection. Mesenteric lymph node (LN) cells were cultured without (medium) or with Trichuris antigen for $72 \mathrm{~h}$ and cell-free supernatants were analyzed by enzyme-linked immunosorbent assay (ELISA) for the expression of IL-12/23p40. (b) Expression of I/12a and I/12b mRNA in the intestinal tissue from Trichuris-infected Ship $1^{\mathrm{F} / \mathrm{F}}$ and Ship $1^{\mathrm{ALysM}}$ mice was examined by quantitative polymerase chain reaction (qPCR). (c) Bone marrow-derived macrophages from Ship $1^{\mathrm{F} / \mathrm{F}}$ and Ship $1^{\Delta \mathrm{L} y s \mathrm{M}}$ mice were stimulated with lipopolysaccharide (LPS) $\left(50 \mathrm{ng} \mathrm{ml}^{-1}\right)$ for $20 \mathrm{~h}$ and cell-free supernatants were analyzed by ELISA for the expression of IL-12/23p40. Data are mean \pm s.e.m. from one experiment and are representative of three independent experiments $(n=12-15$ for panels a and $\mathbf{b}) .{ }^{*} P<0.05$.

study using conventional Ship $1^{-1-}$ mice showed only a modest 1.8-fold increase in the number of Arg1-positive macrophages in the large intestine in the steady state. ${ }^{39}$ Thus, it appears that Ship1 is differentially required for inhibition of M2 macrophage differentiation in a tissue-specific manner.

The role of macrophages during helminth infection has been a matter of controversy. Following infection with $H$. polygyrus, there is a 20-fold increase in Arg1-positive macrophages that are found surrounding tissue-embedded parasites and are critical for parasite expulsion. ${ }^{12,41}$ In contrast, while macrophage numbers significantly increase in the intestine during Trichuris infection, ${ }^{42,43}$ Arg1-positive macrophage numbers do not demonstrate a striking increase. ${ }^{24}$ Further, mice treated with an Arg1 inhibitor (BEC), macrophage-specific Arg1 knockout mice, or mice depleted of macrophages are all resistant to Trichuris infection, demonstrating that macrophages and/or Arg1 activity are not absolutely required for immunity. ${ }^{26}$
However, our results show that if macrophage responses are dysregulated (i.e., increased IL-12 production), immunity to Trichuris is abolished. Thus, there is a critical regulatory role for Ship 1 in the development of type 2 immunity through the inhibition of type 1 responses. These data are reminiscent of previous work in which we show that the cytokine thymic stromal lymphopoietin does not directly promote $\mathrm{T}_{\mathrm{H}} 2$ cell responses, but is required to limit non-protective $\mathrm{T}_{\mathrm{H}} 1$ cell development by blocking IL-12 production. ${ }^{44}$ Thus, vaccines or therapeutic strategies aimed at enhancing $\mathrm{T}_{\mathrm{H}} 2$ cell responses should take the potential negative regulation of type 1 responses into consideration.

There are some instances where macrophages have been shown to play a critical role during helminth infection. Anthony et al. ${ }^{12}$ have demonstrated that macrophages can act as effector cells during infection with the helminth $H$. polygyrus, as depletion of macrophages using clodronate-loaded liposomes resulted in failure to kill tissue-embedded parasites. Furthermore, during infection with S. mansoni, abrogation of M2 macrophage function by macrophage-specific deletion of IL- $4 \mathrm{R} \alpha$ or Arg 1 results in heightened non-protective T-cell responses, severe liver fibrosis, and tissue damage, leading to increased mortality. ${ }^{21,45}$ These results suggest that macrophages play a critical role in certain helminth infections primarily to prevent tissue damage and mortality.

The absence of Ship1 enzymatic activity results in increased levels of PI $(3,4,5) \mathrm{P} 3$ that could lead to increased activation of the PI3K pathway. There are many conflicting reports regarding whether the PI3K pathway has a positive or negative role in TLR-induced production of IL-12/23 and other proinflammatory cytokines. ${ }^{46,47}$ Deletion of the PI3K regulatory subunit p $85 \alpha$ resulted in increased IL- 12 production by DCs. ${ }^{48}$ In contrast, recent studies specifically addressing the role of the PI $3 \mathrm{~K}$ p $110 \alpha$ and $\mathrm{p} 110 \beta$ isoforms in LPS-induced IL-12 production suggest that the PI3K pathway positively regulates IL-12 production. ${ }^{49,50}$ In addition, infection of Ship $1^{-1-}$ BMMacs with Francisella novicida resulted in increased expression of IL-12/23p40 associated with heightened levels of phosphorylated Akt, a marker for activation of the PI3K pathway. ${ }^{51}$ Thus, our results are consistent with these latter studies and suggest that Ship 1 is a negative regulator of IL-12 production, possibly by regulating the levels of $\mathrm{PI}(3,4,5) \mathrm{P} 3$.

In this study, we have used LysM-cre transgenic mice to delete Ship1 in myeloid cells, including macrophages. However, as LysM is also expressed in neutrophils, this cell type may also be playing a role in heightened IL-12 responses and increased susceptibility to Trichuris infection. Future studies using mice with more specific targeted Cre expression will identify whether Ship 1 plays a role in macrophages, neutrophils, or both cell types.

In summary, our results identify a previously unappreciated role for macrophages in immunity to Trichuris. This study suggests that macrophages are not passive players in the development of protective type 2 responses, but can govern the outcome of an infection through modulation of the type of immune response. Future studies examining how Ship1 

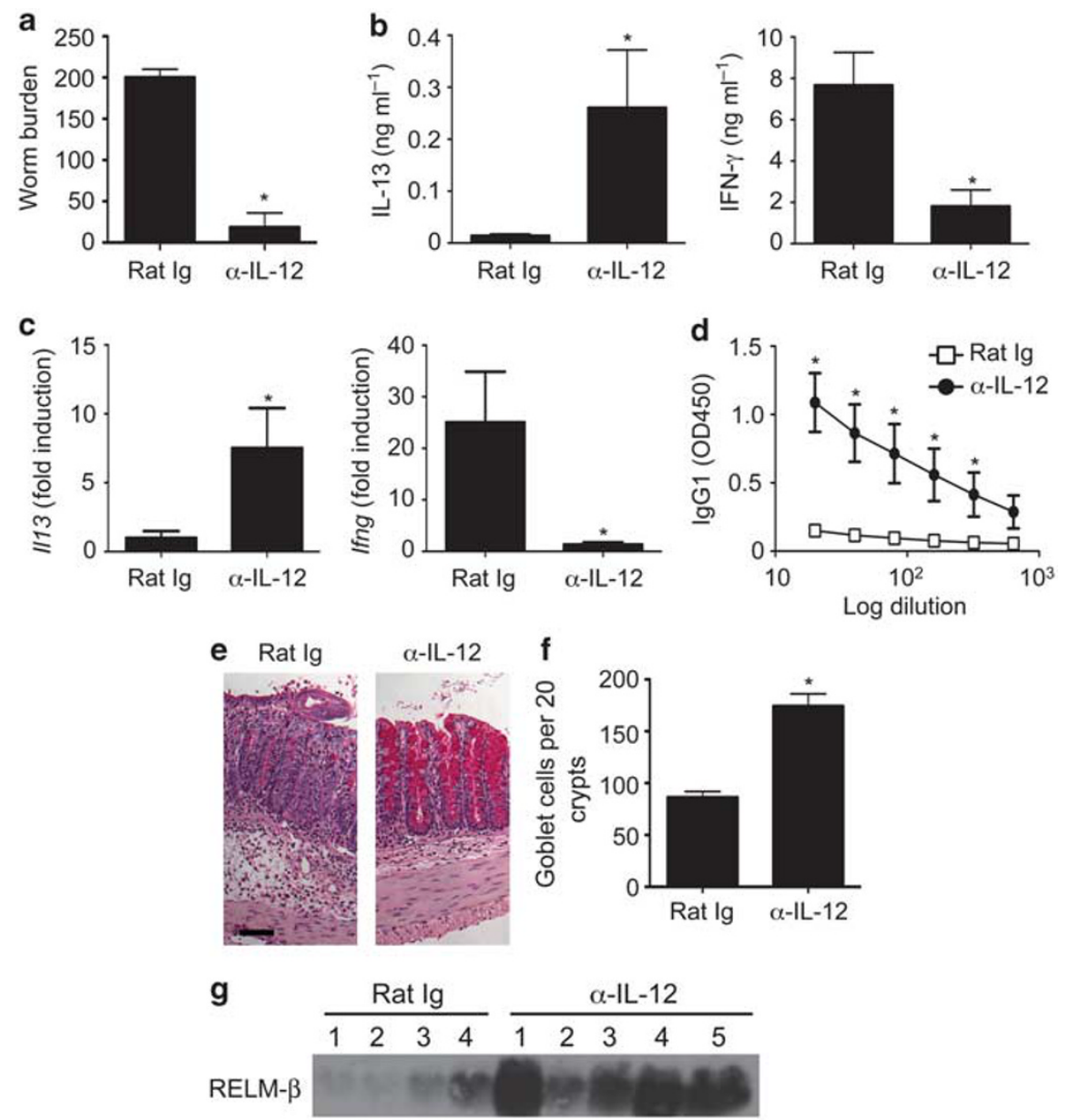

$\begin{array}{llllllllll}1.0 & 1.0 & 1.1 & 1.5 & 4.0 & 1.7 & 2.3 & 3.1 & 2.9\end{array}$

Figure 6 Antibody blockade of interleukin (IL)-12/23p40 in Ship $1^{\Delta L y s \mathrm{M}}$ mice results in increased type 2 immunity and resistance to Trichuris infection. Ship $1^{\Delta \mathrm{LysM}}$ mice were infected with 250 Trichuris eggs, treated intraperitoneally (i.p.) with $500 \mu \mathrm{g}$ of either control rat Ig or anti-IL-12/23p40 (C17.8) every 4 days from day 4 to 20 and killed on day 21 post-infection. (a) Worm burdens were determined microscopically. (b) Mesenteric lymph node (LN) cells were cultured with Trichuris antigen for $72 \mathrm{~h}$ and cell-free supernatants were analyzed by enzyme-linked immunosorbent assay (ELISA) for the expression of IL-13 and interferon (IFN)- $\gamma$. (c) //13 and Ifng mRNA expression in intestinal tissues isolated from rat Ig and $\alpha$-IL-12/23p40-treated Trichuris-infected Ship $1^{\Delta \mathrm{LySM}}$ mice were examined by quantitative polymerase chain reaction (qPCR). (d) Trichuris-specific serum immunoglobulin responses were determined by ELISA. (e) Cecal sections were stained with periodic acid-Schiff's stain. Bar $=25 \mu \mathrm{M}$. (f) Goblet cells were enumerated microscopically. (g) Immunoblot for resistin-like molecule (RELM) $\beta$ secreted into the gut lumen with relative densitometry values. Data are mean \pm s.e.m. and are from one experiment representative of three independent experiments $\left(n=4-5\right.$ per experiment). ${ }^{*} P<0.05$. Ship 1 , SH2-containing inositol 5'-phosphatase 1.

negatively regulates IL-12 expression will shed light on potential novel regulatory mechanisms that control immunity and inflammation.

\section{METHODS}

Mice. LysM-Cre mice were obtained from The Jackson Laboratory (Bar Harbor, ME). The conditional Ship1 allele was generated as described previously. ${ }^{52}$ We observed no Cre toxicity as Cre ${ }^{+}$Ship $1^{\mathrm{F} /+}$ mice were resistant to infection. Animals were maintained in a specific pathogenfree environment and tested negative for pathogens in routine screening. All experiments were carried out at the University of British Columbia following institutional and Canadian Council on Animal Care (CCAC) guidelines approved by the UBC Committee on Animal Care.

Parasites and infections. Isolation of Trichuris excretory-secretory antigen and eggs was carried out as described previously. ${ }^{53}$ Mice were infected on day 0 with 200-250 embryonated eggs, and parasite burdens were assessed microscopically on day 21 or 32 post-infection.

Analysis of Trichuris-induced immunity. Single-cell suspensions from $\mathrm{mLN}$ of naïve or Trichuris-infected mice were plated at $3-4 \times 10^{6}$ per $\mathrm{ml}$ in the medium or in the presence of antibodies against CD3 (145-2C11) and CD28 (37.51; $1 \mu \mathrm{g} \mathrm{ml}^{-1}$ each; eBioscience, San Diego, CA) or Trichuris antigen $\left(50 \mu \mathrm{g} \mathrm{ml}^{-1}\right)$ for $72 \mathrm{~h}$. Cytokine production from cell-free supernatants was determined by standard sandwich enzyme-linked immunosorbent assay (ELISA) using commercially available antibodies (eBioscience). Trichuris-specific serum IgG1 and IgG2a levels were determined by ELISA on plates coated with Trichuris Ag $\left(5 \mu \mathrm{g} \mathrm{ml}^{-1}\right)$. Total protein was isolated from fecal samples, resolved by sodium dodecyl sulfatepolyacrylamide gel electrophoresis, and immunoblotted using a rabbit anti-mouse RELM $\beta$ antibody (PeproTech, Rocky Hill, NJ). ${ }^{2}$ Cecal tissue samples were fixed in $4 \%$ paraformaldehyde and paraffin-embedded. Tissue sections were stained with periodic acid-Schiff (PAS) for visualization 
of goblet cells. Slides were analyzed on a Zeiss Axioplan2 microscope and images captured using a Qimaging Retiga EX CCD camera and the Openlab 4.0.4 software (Perkin-Elmer, Waltham, MA).

Quantitative RT-PCR. mRNA was isolated from tissue or cell samples using TRIzol according to the manufacturer's instructions Invitrogen (Grand Island, NY). Reverse transcription was used to generate cDNA and quantitative polymerase chain reaction was performed using SYBR green (Fermentas, Burlington, ON). The primers used for quantitative polymerase chain reaction analysis were the following: Actb forward, $5^{\prime}$-ACTAATGGCAACGAGCGGTTC-3' and reverse, 5' -GGATGCCACAGGATTCCATACC-3'; Arg1 forward, $5^{\prime}$-CAGAAGAATGGAAGAGTCAG-3' ${ }^{\prime}$ and reverse, $5^{\prime}$-CAGATATG CAGGGAGTCACC-3'; Ship1 forward, 5'-ACTCTGCGTGCTG TTCCGGA-3' and reverse, 5'-CCTTCGGATGCCTGAACAGTG-3'; Nos 2 forward, $5^{\prime}$-CGAAACGCTTCACTTCCAA- $3^{\prime}$ and reverse, $5^{\prime}$ TGAGCCTATATTGCTGTGGCT- $3^{\prime}$. All other primers were purchased from Qiagen (Germantown, MD). Reactions were carried out in an ABI 7900 real-time PCR machine (Applied Biosystems, Carlsbad, CA). Values are expressed relative to $\beta$-actin.

Bone marrow-derived macrophages. BMMacs were generated by culturing bone marrow cells $\left(1 \times 10^{6}\right.$ cells per $\left.\mathrm{ml}\right)$ with recombinant macrophage colony-stimulating factor $\left(10 \mathrm{ng} \mathrm{ml}^{-1}\right.$; StemCell Technologies, Vancouver, BC) for 8 days. On day 8 , macrophages were harvested and plated at $5 \times 10^{6}$ cells per $\mathrm{ml}$ and stimulated with LPS ( $50 \mathrm{ng} \mathrm{ml}^{-1}$; Sigma, St Louis, MO) for $24 \mathrm{~h}$. Cell-free supernatants were collected and analyzed by ELISA.

In vivo antibody treatment. Monoclonal antibodies against IFN- $\gamma$ (XMG-1.2) and IL-12/23p40 (C17.8) were purchased from Bio-X-Cell (West Lebanon, $\mathrm{NH}$ ). Mice were treated intraperitoneally with $1 \mathrm{mg}$ of antibody every 4 days between days 4 and 20 post-infection.

Statistics. Results represent mean \pm s.e.m. Statistical significance was determined by Student's $t$-test using Prism 4.0 (GraphPad Software, La Jolla, CA).

SUPPLEMENTARY MATERIAL is linked to the online version of the paper at http://www.nature.com/mi

\section{ACKNOWLEDGMENTS}

We thank Dr Ken Harder for critical reading of the manuscript. This work was supported by the Canadian Institutes of Health Research (MOP89773 and MOP-106623 to C.Z. and MOP-93580 and MOP-84545 to K.M.M.), Canada Foundation for Innovation (to C.Z.), the National Institutes of Health (RO1 HL72523, RO1 HL085580, RO1 HL107127-01 to W.G.K.), and the Paige Arnold Butterfly Run (to W.G.K.). F.A. is the recipient of a ClHR/Canadian Association of Gastroenterology/Crohn's and Colitis Foundation of Canada postdoctoral fellowship. W.G.K. is the Murphy Family Professor of Children's Oncology Research; K.M.M. is a Michael Smith Foundation for Health Research Senior Scholar; and C.Z. is a CIHR New Investigator and a MSFHR Career Investigator.

\section{DISCLOSURE}

The authors declared no conflict of interest.

(C) 2012 Society for Mucosal Immunology

\section{REFERENCES}

1. Finkelman, F.D. et al. Cytokine regulation of host defense against parasitic gastrointestinal nematodes: lessons from studies with rodent models. Annu. Rev. Immunol. 15, 505-533 (1997).

2. Artis, D. et al. RELMbeta/FIZZ2 is a goblet cell-specific immune-effector molecule in the gastrointestinal tract. Proc. Natl. Acad. Sci. USA 101, 13596-13600 (2004).
3. Datta, R. et al. Identification of novel genes in intestinal tissue that are regulated after infection with an intestinal nematode parasite. Infect. Immun. 73, 4025-4033 (2005).

4. Cliffe, L.J. et al. Accelerated intestinal epithelial cell turnover: a new mechanism of parasite expulsion. Science 308, 1463-1465 (2005).

5. Khan, W.I. et al. Modulation of intestinal muscle contraction by interleukin9 (IL-9) or IL-9 neutralization: correlation with worm expulsion in murine nematode infections. Infect. Immun. 71, 2430-2438 (2003).

6. Akiho, H., Blennerhassett, P., Deng, Y. \& Collins, S.M. Role of IL-4, IL-13, and STAT6 in inflammation-induced hypercontractility of murine smooth muscle cells. Am. J. Physiol. Gastrointest. Liver Physiol. 282, G226-G232 (2002).

7. Else, K.J., Finkelman, F.D., Maliszewski, C.R. \& Grencis, R.K. Cytokinemediated regulation of chronic intestinal helminth infection. J. Exp. Med. 179, 347-351 (1994)

8. Helmby, H., Takeda, K., Akira, S. \& Grencis, R.K. Interleukin (IL)-18 promotes the development of chronic gastrointestinal helminth infection by downregulating IL-13. J. Exp. Med. 194, 355-364 (2001).

9. Mosser, D.M. \& Edwards, J.P. Exploring the full spectrum of macrophage activation. Nat. Rev. Immunol. 8, 958-969 (2008).

10. Unanue, E.R. Antigen-presenting function of the macrophage. Annu. Rev. Immunol. 2, 395-428 (1984).

11. MacMicking, J., Xie, Q. \& Nathan, C. Nitric oxide and macrophage function. Annu. Rev. Immunol. 15, 323-350 (1997).

12. Anthony, R.M. et al. Memory $\mathrm{T}(\mathrm{H}) 2$ cells induce alternatively activated macrophages to mediate protection against nematode parasites. Nat. Med. 12, 955-960 (2006).

13. deSchoolmeester, M.L., Manku, H. \& Else, K.J. The innate immune responses of colonic epithelial cells to Trichuris muris are similar in mouse strains that develop a type 1 or type 2 adaptive immune response. Infect. Immun. 74, 6280-6286 (2006).

14. Reece, J.J. et al. Hookworm-induced persistent changes to the immunological environment of the lung. Infect. Immun. 76, 3511-3524 (2008).

15. MacDonald, A.S., Loke, P., Martynoga, R., Dransfield, I. \& Allen, J.E. Cytokine-dependent inflammatory cell recruitment patterns in the peritoneal cavity of mice exposed to the parasitic nematode Brugia malayi. Med. Microbiol. Immunol. 192, 33-40 (2003).

16. Jenkins, S.J. et al. Local macrophage proliferation, rather than recruitment from the blood, is a signature of TH2 inflammation. Science $\mathbf{3 3 2}$, 1284-1288.

17. Martinez, F.O., Helming, L. \& Gordon, S. Alternative activation of macrophages: an immunologic functional perspective. Annu. Rev. Immunol. 27, 451-483 (2009).

18. Gordon, S. \& Martinez, F.O. Alternative activation of macrophages: mechanism and functions. Immunity 32, 593-604.

19. Gordon, S. Alternative activation of macrophages. Nat. Rev. Immunol. 3 , 23-35 (2003)

20. Rodriguez, P.C. et al. -Arginine consumption by macrophages modulates the expression of CD3 zeta chain in T lymphocytes. J. Immunol. 171, 1232-1239 (2003).

21. Herbert, D.R. et al. Alternative macrophage activation is essential for survival during schistosomiasis and downmodulates Thelper 1 responses and immunopathology. Immunity 20, 623-635 (2004).

22. Michels, C.E. et al. Neither interleukin-4 receptor alpha expression on CD4+ T cells, or macrophages and neutrophils is required for protective immunity to Trichinella spiralis. Immunology 128, e385-394 (2009).

23. Herbert, D.R. et al. Arginase I suppresses IL-12/IL-23p40-driven intestinal inflammation during acute schistosomiasis. J. Immunol. 184, 6438-6446 (2010).

24. Bowcutt, R. et al. Arginase-1-expressing macrophages are dispensable for resistance to infection with the gastrointestinal helminth Trichuris muris. Parasite Immunol. 33, 411-420 (2011).

25. Cliffe, L.J. \& Grencis, R.K. The Trichuris muris system: a paradigm of resistance and susceptibility to intestinal nematode infection. Adv. Parasitol. 57, 255-307 (2004).

26. Perrigoue, J.G. et al. MHC class II-dependent basophil-CD4+ T cell interactions promote $\mathrm{T}(\mathrm{H}) 2$ cytokine-dependent immunity. Nat. Immunol. 10, 697-705 (2009).

27. Haddon, D.J. et al. SHIP1 is a repressor of mast cell hyperplasia, cytokine production, and allergic inflammation in vivo. J. Immunol. 183, 228-236 (2009). 
28. Helgason, C.D. et al. Targeted disruption of SHIP leads to hemopoietic perturbations, lung pathology, and a shortened life span. Genes Dev. 12, 1610-1620 (1998).

29. Ghansah, T. et al. Expansion of myeloid suppressor cells in SHIP-deficient mice represses allogeneic T cell responses. J. Immunol. 173, 7324-7330 (2004).

30. Antignano, F. et al. SHIP is required for dendritic cell maturation. J. Immunol. 184, 2805-2813 (2011).

31. Collazo, M.M. et al. SHIP limits immunoregulatory capacity in the T-cell compartment. Blood 113, 2934-2944 (2009).

32. Kashiwada, M. et al. Downstream of tyrosine kinases-1 and Src homology 2-containing inositol $5^{\prime}$-phosphatase are required for regulation of CD4+CD25+ T cell development. J. Immunol. 176, 3958-3965 (2006).

33. Kuroda, E. et al. SHIP represses Th2 skewing by inhibiting IL-4 production from basophils. J. Immunol. 186, 323-332 (2011).

34. Rauh, M.J. et al. SHIP represses the generation of alternatively activated macrophages. Immunity 23, 361-374 (2005).

35. Sly, L.M., Rauh, M.J., Kalesnikoff, J., Song, C.H. \& Krystal, G. LPS-induced upregulation of SHIP is essential for endotoxin tolerance. Immunity $\mathbf{2 1}$, 227-239 (2004).

36. Clausen, B.E., Burkhardt, C., Reith, W., Renkawitz, R. \& Forster, I. Conditional gene targeting in macrophages and granulocytes using LysMcre mice. Transgenic Res. 8, 265-277 (1999).

37. Malvin, N.P., Seno, H. \& Stappenbeck, T.S. Colonic epithelial response to injury requires Myd88 signaling in myeloid cells. Mucosal Immunol. 5, 194-206 (2012).

38. Ishikawa, T.O., Oshima, M. \& Herschman, H.R. Cox-2 deletion in myeloid and endothelial cells, but not in epithelial cells, exacerbates murine colitis. Carcinogenesis 32, 417-426 (2011).

39. Weisser, S.B. et al. SHIP-deficient, alternatively activated macrophages protect mice during DSS-induced colitis. J. Leukoc. Biol. 90, 483-492 (2011).

40. Reece, J.J., Siracusa, M.C. \& Scott, A.L. Innate immune responses to lung-stage helminth infection induce alternatively activated alveolar macrophages. Infect. Immun. 74, 4970-4981 (2006).
41. Sugawara, Y., Azuma, N., Onodera, S., Tsunoka, Y. \& Morimoto, M. Th2 immune responses and alternatively activated macrophages (AAMacs) in helminth infection in aged mice. J. Vet. Med. Sci. 73, 511-516 (2011).

42. deSchoolmeester, M.L., Little, M.C., Rollins, B.J. \& Else, K.J. Absence of CC chemokine ligand 2 results in an altered Th1/Th2 cytokine balance and failure to expel Trichuris muris infection. J. Immunol. 170, 4693-4700 (2003).

43. Little, M.C., Bell, L.V., Cliffe, L.J. \& Else, K.J. The characterization of intraepithelial lymphocytes, lamina propria leukocytes, and isolated lymphoid follicles in the large intestine of mice infected with the intestinal nematode parasite Trichuris muris. J. Immunol. 175, 6713-6722 (2005).

44. Zaph, C. et al. Epithelial-cell-intrinsic IKK-beta expression regulates intestinal immune homeostasis. Nature 446, 552-556 (2007).

45. Pesce, J.T. et al. Arginase-1-expressing macrophages suppress Th2 cytokine-driven inflammation and fibrosis. PLoS Pathog. 5, e1000371 (2009).

46. Hazeki, K., Nigorikawa, K. \& Hazeki, O. Role of phosphoinositide 3-kinase in innate immunity. Biol. Pharm. Bull. 30, 1617-1623 (2007).

47. Fukao, T. \& Koyasu, S. PI3K and negative regulation of TLR signaling. Trends Immunol. 24, 358-363 (2003).

48. Fukao, T. et al. PI3K-mediated negative feedback regulation of IL-12 production in DCs. Nat. Immunol. 3, 875-881 (2002).

49. Utsugi, M. et al. PI3K p110beta positively regulates lipopolysaccharideinduced IL-12 production in human macrophages and dendritic cells and JNK1 plays a novel role. J. Immunol. 182, 5225-5231 (2009).

50. Utsugi, M. et al. JNK1 and JNK2 differently regulate IL-12 production in THP-1 macrophage cells. Cytokine 51, 127-131 (2010).

51. Parsa, K.V. et al. Macrophage pro-inflammatory response to Francisella novicida infection is regulated by SHIP. PLoS Pathog. 2, e71 (2006).

52. Wang, J.W. et al. Influence of SHIP on the NK repertoire and allogeneic bone marrow transplantation. Science 295, 2094-2097 (2002).

53. Antignano, F., Mullaly, S.C., Burrows, K. \& Zaph, C. Trichuris muris infection: a model of type 2 immunity and inflammation in the gut. J. Vis. Exp. 24, pii 2774 (2011). 